\title{
Uferfiltration - Stand der Technik und neue Herausforderungen
}

\author{
Sebastian Handl · Christoph Schmoller · Reinhard Perfler
}

Angenommen: 1. September 2020 / Online publiziert: 6. Oktober 2020

(c) Der/die Autor(en) 2020

\begin{abstract}
Zusammenfassung Die Uferfiltration ist eine naturnahe Methode der Trinkwassergewinnung, bei der Oberflächenwasser durch die Förderung von ufernahem Grundwasser in den Untergrund infiltriert und dabei natürlich gereinigt wird. Während der Fließstrecke vom Oberflächenwasser bis hin zum Brunnen finden verschiedene physikalische, chemische und mikrobiologische Prozesse statt. Neben der Aufenthaltszeit des Wassers im Untergrund haben die Redoxbedingungen den größten Einfluss auf wesentliche Entfernungsvorgänge wie biologischen Abbau und Sorption. Neben den noch unspezifischen Konsequenzen infolge des Kli-

the process naturally purified. Along the way from surface water to the well, physical, chemical and microbiologic procedures take place. Besides the water residence time in the subsoil the redox-conditions have the greatest effect on significant removal processes like biological degradation or sorption. Besides unspecific consequences due to climate change, nowadays, emerging pollutants, which partly are insufficiently studied and toxicologically difficult to predict, constitute the main challenge. Bank filtration is an effective method to reduce costs in comparison to alternative treatment methods for surface water.
\end{abstract} mawandels, stellen heute insbesondere "neu aufkommende“ Spurenstoffe, die teilweise noch unzureichend erforscht und in toxikologischer Hinsicht schwer einschätzbar sind, das zentrale Problem dar. Die Uferfiltration stellt im Vergleich zu alternativen Aufbereitungsverfahren von Wasser aus Oberflächengewässern eine effektive Methode insbesondere auch zur Reduktion der Kosten dar.

Schlüsselwörter Uferfiltration .

Untergrundpassage .

Trinkwasseraufbereitung .

Reinigungsprozesse $\cdot$ Naturnahe Systeme

\section{Bank filtration - state of the art and new challenges}

Abstract Bank filtration is a natural method of drinking water abstraction. Surface water is infiltrated into the subsoil by extracting groundwater and in

DI S. Handl (四) · C. Schmoller . PD DI Dr. R. Perfler

Department Wasser-Atmosphäre-

Umwelt, Institut für

Siedlungswasserbau, Industriewasserwirtschaft und

Gewässerschutz, Universität

für Bodenkultur Wien,

Muthgasse 18, 1190 Wien, Österreich

sebastian.handl@boku.ac.at
Keywords Bank filtration

Underground passage $\cdot$ Drinking water treatment - Purification processes .

Nature-based solutions

\section{Einleitung}

Die Uferfiltration ist die Gewinnung von Wasser aus einem oberirdischen Gewässer, das aufgrund der Förderung von ufernahem Grundwasser in den Untergrund infiltriert und dabei natürlich gereinigt wird. Die Uferfiltration nimmt als Ressource eine Zwischenstellung zwischen Grundwasser und Oberflächenwasser ein. In Bezug auf die quantitative Verfügbarkeit ähnelt sie den Oberflächengewässern und ist weit über jener von Brunnen in reinem Grundwasser anzusiedeln. Während der Uferpassage finden einerseits Reinigungsprozesse und andererseits Mischung mit landseitigem Wasser statt. Dadurch werden Schadstoffe abgebaut, kurzfristige Änderungen der Wasserqualität gedämpft (Vergleichmäßigung der Wasserqualität im zeitlichen Verlauf) und insbesondere wird die chemische Stabilität erhöht (Lee et al. 2009; zit. in Ahmed und Marhaba 2016). Diese Vorteile führen dazu, dass die Uferfiltration mehr und mehr an Bedeutung gewinnt, sowohl für die Trinkwasserver- sorgung als auch für die Nutzwasserbereitstellung (Kühl- und Prozesswasser).

Schmoller (2014) und Ahmed und Marhaba (2016) geben einen Überblick zum Stand des Wissens in der Literatur über die beteiligten Prozesse und Einflussfaktoren bei der Uferfiltration. Richardson und Kimura (2020) stellen aktuelle wissenschaftliche Entwicklungen bei der Untersuchung von Spurenstoffen dar. Diese drei Publikationen werden im vorliegenden Artikel besonders berücksichtigt.

\section{Reinigungsvorgänge bei der Bodenpassage}

Bei der Reinigung von Oberflächenwasser durch Uferfiltration wirken vier wesentliche Mechanismen: mechanische Filtration, Sedimentation, Sorption und mikrobiologischer Abbau. Die beiden Prozesse mechanische Filtration und Sedimentation sind wichtig zur Partikelentfernung insbesondere während der Infiltration. Sorptive Kräfte spielen eine wichtige Rolle bei der Abscheidung von partikulären Schadstoffen und Mikroorganismen während der Untergrundpassage. Stoffe, die bereits an der Bodenmatrix sorbiert sind, können häufig besser mikrobiologisch abgebaut werden. Gelöste organische Substanzen und auch kleine partikelartige Stoffe werden in Abhängigkeit vom Verbrauch an Elektronen-Akzeptoren (Sauerstoff, Mangan, Eisen, Nitrat, Sulfat) sowie der möglichen Veränderung der Redoxverhältnisse und des $\mathrm{pH}$-Werts vor allem durch die Aktivität von Mikroorganismen abgebaut (Schulte-Ebbert 2004). In mehreren verschiedenen Untersuchungen wurde festgestellt, dass die biologische Aktivität mit zunehmender Filtertiefe abnimmt (Abb. 1).

Sontheimer et al. (1981; zit. in Ziegler 2001) stellen eine gute Wirkung der Bodenpassage bei der Filtrierung von Partikeln, Kolloiden und pathogenen Keimen fest. Die Entfernung von Ionen und hydrophoben organischen Stoffen führen sie auf Fällungsprozes- 


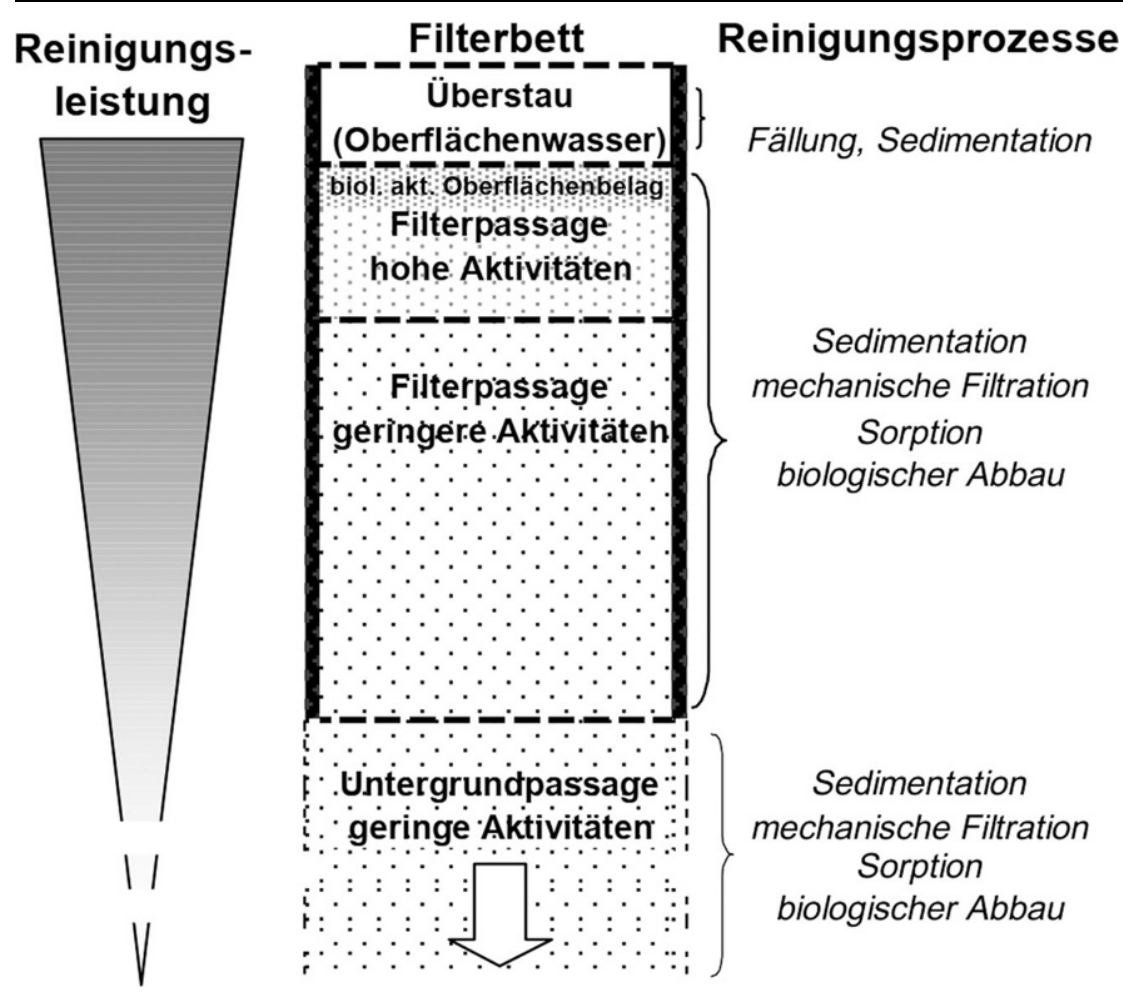

Abb. 1 Reinigungsprozesse während der Infiltration und Untergrundpassage (nach Preuß und Schulte-Ebbert 2000; zit. in Schulte-Ebbert 2004)

se und Sorption zurück. Biologischer Abbau wird als wesentlicher Wirkmechanismus bei der Entfernung von Ammonium und abbaubaren organischen Wasserinhaltsstoffen sowie bei der Inaktivierung von Keimen und Viren identifiziert. Dieser Prozess ist bei konservativen Ionen (z. B. Chlorid) und polaren, biologisch schwer abbaubaren organischen Substanzen nicht wirksam. Die wesentlichen Einflussfaktoren stellen die Redoxbedingungen im Untergrund, die Qualität des infiltrierenden Wassers und des natürlichen Grundwassers, die Fließstrecke, die Fließdauer, die Bodenbeschaffenheit sowie die klimatischen Verhältnisse dar (Ziegler 2001).

Viele Reinigungsprozesse gehen mit einem Abbau des gelösten Sauerstoffs im Wasser einher. Dadurch können auch anoxische Bedingungen auftreten, in deren Folge Denitrifikation auftritt, aber auch Sulfat, Eisen (III) und Mangan (VI) reduziert werden können (Massmann et al. 2008; zit. in. Ahmed und Marhaba 2016). Diem et al. (2013; zit. in. Ahmed und Marhaba 2016) stellen ein Modell vor, das die Vorhersage des Sauerstoffgehalts in Abhängigkeit von der Distanz zum Fluss erlaubt. Dabei werden insbesondere die Ausgangskonzentrationen im Ober- flächengewässer berücksichtigt, aber auch hydrologische und klimatische Randbedingungen.

\subsection{Partikuläre Wasserinhaltsstoffe}

Partikuläre Stoffe sind praktisch in allen Oberflächenwässern enthalten. Sie können bis zu Größenordnungen von 50-500 nm im Grundwasser transportiert werden. Der Partikelrückhalt bei der Uferfiltration ist sowohl auf Oberflächen- als auch auf Tiefenfiltration zurückzuführen (Ziegler 2001).

An den partikulären Soffen können sich sowohl anorganische Schadstoffe wie Spurenmetalle, als auch organische Verunreinigungen (z. B. unpolare Stoffe) und mikrobiologische Verunreinigungen (Bakterien, Viren, Protozoen und Parasiten) anhaften. Deswegen ist für eine weitestgehende Entfernung der partikulären Stoffe zu sorgen. Dabei spielen die Porenwassergeschwindigkeit und Einflüsse auf die elektrochemischen Eigenschaften der Partikel eine wesentliche Rolle. Zum Beispiel kann durch eine Veränderung des pH-Werts und der damit einhergehenden Verringerung des Oberflächenpotenzials an Feststoffen eine Agglomeration dieser Feststoffe hervorgerufen werden. Ande- rerseits kann auch eine Destabilisierung bereits gebildeter Aggregate durch eine höhere Ionenstärke ausgelöst werden. Weiters kann die Sorption von Huminstoffen wesentlich die Transportreichweite von Partikel im Untergrund erhöhen. Generell ist das Abscheidepotenzial der Untergrundpassage in Bezug auf suspendierte oder kolloidale stabile Partikel hoch. Großteils werden in Uferfiltraten Trübstoffgehalte unter 0,1 FNU gemessen. (Hofmann und Schöttler 1998; Schulte-Ebbert 2004). Die Effektivität der Untergrundpassage bei der Entfernung von Trübstoffen wird auch in vielen internationalen Studien bestätigt. Die Trübung wird dabei um mehr als eine log-Stufe reduziert (Mikels 1992; Dash et al. 2010; Weiss et al. 2005; zit. in Ahmed und Marhaba 2016).

\subsection{Mikroorganismen und Viren}

Filtrations- und Sorptionsprozess sowie biologische Inaktivierung sind für die Entfernung von Mikroorganismen (Bakterien, Protozoen, Algen etc.) und Viren bei der Uferfiltration verantwortlich. Den entscheidenden Abschnitt zur Elimination von Viren und Bakterien stellt dabei die Bodenpassage dar (Botzenhart 1998; zit. in Ziegler 2001).

Bakterien werden in den obersten Zentimetern des Filters durch Adsorption effektiv zurückgehalten. Dieser Bereich ist auch durch eine äußerst aktive Mikrofauna und -flora gekennzeichnet. Weiters spielen die Sorption von Stoffen an organischen und anorganischen Oberflächen und die mechanische Filtration in tieferen Schichten von Sandfiltern und bei der Untergrundpassage eine wichtige Rolle. Modelluntersuchungen zeigen, dass die Transportreichweite von Bakterien durch größere Filterkorndurchmesser erhöht wird (Ellis und Aydin 1995; zit. in Schulte-Ebbert 2004).

Lahti et al. (1996; zit. in Schulte-Ebbert 2004) stellen fest, dass Cyanobakterien und ihre Toxine bei Filtration durch sandige Sedimente oder Böden durch Adsorption und biologischen Abbau effektiv entfernt werden.

Protozoen werden aufgrund ihrer Größe (einige Mikrometer) effektiv durch Filtration eliminiert. (Schubert 2000; zit. in Ziegler 2001).

Ahmed und Marhaba (2016) fassen mehrere Untersuchungen von Dash et al. (2010), Ghodeif et al. (2016), Shamrukh und Abdel-Wahab (2011), 
Singh et al. (2010) und Weiss et al. (2005) an den Flüssen Ohio, Wabash, Missouri (alle USA), Nil (Ägypten), Ganges und Yamuna (Indien) zusammen. Im Großteil der Untersuchungsgebiete, vor allem bei geringeren Belastungen, werden coliforme Bakterien und fäkalcoliforme Bakterien komplett entfernt. In den übrigen Untersuchungen (Nil, Ganges und Yamuna), in denen die auch Belastungen deutlich höher liegen, werden Reduktionen im Bereich von 2 bis 4 log-Stufen berichtet.

Dash et al. (2008; zit. in Ahmed und Marhaba 2016) vergleichen Uferfiltration mit Schnellsandfiltern und stellen 5,2 bzw. 4,2 log-Stufen Reduktion von coliformen bzw. fäkalcoliformen Bakterien bei der Uferfiltration fest. Wohingegen bei der Schnellsandfiltration lediglich 4,2 log-Stufen Reduktion berichtet werden.

Wang (2005; zit. in Ahmed und Marhaba 2016) weist darauf hin, dass die Reduktion von mikrobieller Kontamination durch die Uferfiltration in weiterer Folge auch das Risiko der Bildung von problematischen Nebenprodukten, die bei der Desinfektion (DBP - disinfection by-products) entstehen können, verringert wird.

\subsection{Nährstoffe}

Gewässer in Regionen mit intensiver Landwirtschaft oder mit hohen Anteilen an Kläranlagenabläufen weisen teilweise hohe Nährstoffkonzentrationen von Ammonium, Nitrat und Phosphat auf. Bei der Trinkwassergewinnung durch Uferfiltration stellen sie in der Regel jedoch kein Problem dar (Ziegler 2001).

Nitrifikation führt unter oxischen Bedingungen im Untergrund zur Umwandlung von Ammonium zu Nitrat. Dieser Prozess führt jedoch schon bei geringen Mengen an Ammonium zu einer ausgeprägten Sauerstoffzehrung (4,6g $\mathrm{O}_{2} / \mathrm{g} \quad \mathrm{NH}_{4}-\mathrm{N}$ ) (Schachtschabel et al. 1998; zit. in Ziegler 2001).

Doussan et al. (1998; zit. in Ziegler 2001) weisen auf die hohe Adsorbierbarkeit von Ammonium an Kalk hin. Bei reduzierenden Bedingungen (z. B. infolge von hohen Organikfrachten) kann Ammonium aber teilweise auch den Boden passieren. Allerdings kommt es erst unter anoxischen Bedingungen und bei Vorliegen abbaubarer organischer Kohlenstoffverbindungen zur Denitrifikation von vorhandenem Nitrat (Ziegler 2001).
Der gute Rückhalt von Phosphat unter neutralen bis alkalischen Bedingungen ist auf die Bildung schwerlöslicher Calcium-, Eisen- oder Aluminiumphosphate zurückzuführen. Driescher et al. (1989; zit. in Ziegler 2001) konnten allerdings eine erhebliche Phosphatbeweglichkeit bei der Uferfiltration am Müggelsee nachweisen.

\subsection{Schwermetalle und Metallkationen}

Den Hauptprozess zur Entfernung von Eisen, Mangan und verschiedenen Schwermetallen stellt die Sorption dar, wobei diese stark vom $\mathrm{pH}$-Wert beeinflusst ist (Schöttler 1986; zit. in Ziegler 2001). Im aeroben Bereich findet vor allem Ionenaustausch an Tonmineralien, Eisen- und Aluminiumoxiden oder organischen Feststoffen statt. Unter anoxischen Bedingungen dominieren Fällungsreaktionen mit Sulfid, welches unter oxischen Bedingungen nicht vorhanden ist (Förstner 1995; zit. in Ziegler 2001). Beim Auftreten unterschiedlicher Milieubedingungen können Eliminations- und Remobilisierungsmechanismen auch hintereinander ablaufen (Ziegler 2001).

Stuyfzand und Kooiman (1996; zit. in Schulte-Ebbert 2004) stellten eine effektive Abscheidung zahlreicher Schwermetalle und organischer Schadstoffe mit Ausnahme einiger Pestizide am Rhein fest.

Schöttler (1995; zit. in Schulte-Ebbert 2004) bemerkte hohe Eliminationsraten von Eisen und Mangan in den ersten Zentimetern der Untergrundpassage, wohingegen Kupfer, welches in geringen Konzentrationen auftrat, teilweise auch mobilisiert werden konnte.

Die Eliminierung von Schwermetallen sollte generell nicht alleine durch natürliche bzw. naturnahe Verfahren, wozu er neben der Bodenpassage auch Langsamsandfilter zählt, konzipiert werden, sondern in Kombination mit technischen Lösungen wie Ionenaustausch, Fällung, Filtration und eventuell Komplexierung, wodurch ein zeitlich befristeter aber starker Rückhalt möglich ist. Die Anhäufung von Spurenmetallen kann zur Gefährdung und Verminderung biologischer Prozesse und darüber hinaus, bei geänderten Rohwasser- und Betriebsbedingungen, zur Wiederfreisetzung vereinzelter Spurenmetalle führen. Blei ist bei optimalen Bedingungen sehr gut bis gut durch eine Bodenpassage oder Langsamsandfiltration entfernbar. Die Entfernbarkeit von Cadmium, Chrom, Zink und Cyanid ist als mäßig einzustufen. Für Arsen und Nickel sind die beiden Verfahren nachweislich ungeeignet (Jekel und Haberer 2004).

\subsection{Organische Wasserinhaltsstoffe}

Gelöste organische Wasserinhaltsstoffe umfassen natürliche organische Stoffe (Huminstoffe, niedermolekulare Säuren, Proteine, Kohlenhydrate) sowie verschiedene Einzelstoffe (Pestizide, Industriechemikalien, Tenside, Arzneistoffe, Desinfektionsnebenprodukte). Bei der Uferfiltration erfolgt neben der Konzentrationsverminderung durch Mischen mit landseitigem Grundwasser auch eine Reduktion durch biologische Prozesse und Sorption. (Ziegler 2001).

Sontheimer (1991; zit. in Ziegler 2001) führt Untersuchungen mittels Testfiltern durch und ermittelt dabei eine Abbauleistung von $25-50 \%$ der organischen Stoffe. Da die Konzentration abbaubarer organischer Wasserinhaltsstoffe im infiltrierenden Wasser normalerweise gering ist, wurde festgestellt, dass diese in der Untergrundpassage innerhalb einer Fließstrecke von weniger als $1 \mathrm{~m}$ entfernt werden. Daraus wird der Schluss gezogen, dass die Reduktion des DOC (dissolved organic carbon - gelöster organischer Kohlenstoff) an den untersuchten Wasserwerksstandorten entlang des Rheins durch Verlängerung der Fließstrecke nicht messbar verbessert werden kann.

Ahmed und Marhaba (2016) fassen mehrere Untersuchungen von Clayton (1995), Weiss (2005) und Hoppe-Jones et al. (2010) an amerikanischen Flüssen und der Elbe zusammen und stellen ebenfalls fest, dass sowohl DOC wie auch TOC (total organic carbon - gesamter organischer Kohlenstoff) effektiv durch Uferfiltration reduziert werden können.

Herrschen anaerobe Bedingungen in der Untergrundpassage vor, nimmt der organische Kohlenstoff nur mäßig ab. Wohingegen bei aerober Untergrundpassage (und auch Langsamsandfiltration) Eliminationsraten von ca. $70 \%$ erreicht werden können. Dies ist auf die höhere mikrobielle Aktivität zurückzuführen. Diese umfasst die Stufen der Metabolisierung und Mineralisierung organischer Verbindungen, wobei es zur Bildung von Kohlendioxid (im Wasser frei oder als Hydrogencarbonat vorliegend) kommt, und in kleineren Teilen die Ausgangsstoffe mit einem hö- 
heren Oxidationsgrad gebildet werden. Dadurch kann je nach Pufferkapazität des Wassers der pH-Wert abnehmen. In der Regel ist die Abfolge der mikrobiellen Prozesse durch das Angebot an abbaubaren organischen Verbindungen beschränkt. Der Reihe nach werden Sauerstoff, Nitrat, Eisen, Mangan und Sulfat als Elektronenakzeptoren reduziert. Die mikrobiellen Prozesse die zu einem Abbau organischer Verbindungen führen, finden in Abhängigkeit vom verfügbaren Elektronenakzeptor statt. Dabei werden in dieser Reihenfolge Sauerstoff, Nitrat, Eisen, Mangan und Sulfat als Elektronenakzeptoren reduziert. Bei Abwesenheit dieser Elektronenakzeptoren kann es in seltenen Fällen auch zur Methanogenese kommen (Schulte-Ebbert 2004; Preuß und Nehrkorn 1988; Ziegler 2001).

Die organischen Wasserinhaltsstoffe, die den DOC bilden, adsorbieren an Eisen- und Aluminiumoxid sowie in geringerem Ausmaß auch an Kaolinit und Tonbestandteilen des Bodens. Im neutralen pH-Bereich findet keine Sorption an Quarzmineralien statt (Ziegler 2001; Davis 1982; Jardine et al. 1989).

Unpolare organische Wasserinhaltsstoffe, wie polycyclische aromatische Kohlenwasserstoffe, sind meist nur in geringen Konzentrationen im infiltrierenden Wasser enthalten, und werden direkt in der Infiltrationszone abgeschieden und dort weiter mikrobiell umgesetzt Haberer et al. (1984; zit. in Ziegler 2001).

Ausfällungs- und Sorptionsprozesse bei der Bodenpassage wirken besonders auf höhermolekulare Bestandteile des DOC. Sie werden in der Folge biologisch oxidiert, wobei es teils zur Desorption niedermolekularer Anteile kommt, und keine Stoffdepots gebildet werden (Sontheimer 1991; Gerlach 1998; Ziegler 2001).

Untersuchungen zum Vergleich der DOC-Entfernung von Kläranlagenabläufen zeigen bei biologisch inaktiven Säulen lediglich eine Entfernungsrate von $10 \%$, wohingegen bei biologisch aktiven Säulen bis zu $54 \%$ erreicht werden. Neben der Qualität des infiltrierenden Wassers und der Mischung mit landseitigem Grundwasser hat der Standort einen starken Einfluss auf die Entfernung des DOC, wodurch nur eingeschränkte örtliche Vergleichbarkeit gegeben ist (Quanrud et al. 1996; Ziegler 2001).

Der Abbau anthropogener organischer Einzelstoffe wie Pharmazeutika,
Haushalts-, Industriechemikalien, Tenside oder Pestizide ist von der Anfangskonzentration und den vorherrschenden Milieubedingungen (Redoxbedingungen) abhängig. Höher chlorierte und stark substituierte aromatische Kohlenwasserstoffe sind nur schwer abbaubar. Als problematisch bei der Uferfiltration werden synthetische organische Komplexbildner, aromatische Sulfonate, aliphatische Amine, Pharmazeutika, Alkylphenole/Bisphenol A sowie Pestizide identifiziert, weil sie nicht oder nur unzureichend entfernt werden können. (Brauch et al. 2000; Ziegler 2001).

Bei der Uferfiltration und ausreichend langen Fließwegen werden natürliche und synthetische Östrogene, Algentoxine sowie Bisphenol A und iso-Nonylphenole hingegen vollständig entfernt bzw. wesentlich reduziert (Wegener et al. 1999; Chorus et al. 1995; Brauch et al. 2000; Ziegler 2001).

\section{Herausforderungen für die Trinkwasserversorgung aus Uferfiltration}

\subsection{Klimawandel}

Unterschiedliche Auswirkungen des Klimawandels beeinflussen sowohl die Menge als auch die Qualität, Aufenthaltszeiten, Redox-Bedingungen und damit die Reinigungsleistung von Uferfiltratstandorten. Hochwässer führen zur Entfernung etwaiger Kolmationsschichten, während Niederwasser infolge von Trockenperioden deren Aufbau begünstigen. Diese Kolmationsschichten verringern einerseits das verfügbare Wasserdargebot in den Uferfiltratstandorten, andererseits verstärken sie den biologischen Abbau von Schadstoffen. Erhöhungen in der Temperatur und die dadurch schneller ablaufenden biologischen und chemischen Prozesse können zu verstärktem Abbau und damit einhergehend zur Verringerung des Sauerstoffgehalts führen. Die Auswirkungen können standortspezifisch sehr unterschiedlich ausfallen wobei die Uferfiltration eine zusätzliche natürliche Barriere gegen Auswirkungen des Klimawandels darstellt (Hiscock und Grischek 2002; Sprenger et al. 2011; Eckert et al. 2008; zit. in Ahmed und Marhaba 2016).

\section{2 „Neue“ wasserrelevante Stoffe}

Zahlreiche neue Stoffe, die jedes Jahr weltweit in die Umwelt gelangen, stellen für einen nachhaltigen Trinkwasserschutz und den Qualitätserhalt der Wasserressourcen eine große Herausforderung dar. In der EU werden im Jahr über 150 Mio. Tonnen gefährliche (z.B. schädliche, giftige, kanzerogene) Chemikalien produziert (Grathwohl et al. 2012).

Richardson und Kimura (2020) fassen 250 aktuelle wissenschaftliche Artikel zusammen, welche sich mit neuen Umweltschadstoffen (emerging environmental pollutants) beschäftigen, die für den Wassersektor relevant sind. Sie teilen diese in die folgenden elf Stoffgruppen ein:

(i) Die Entstehung und Verbreitung antibiotikaresistenter Bakterien und Gene gehen auf den zunehmenden Einsatz von Antibiotika bei der Humanmedizin, aber auch in der Nutztierhaltung zurück. Die wesentliche Eintragsquelle stellen urbane Kläranlagenabläufe dar.

(ii) Künstliche Süßungsmittel werden in vielen Nahrungsmitteln eingesetzt und verhalten sich teilweise in der Natur, aber auch in Kläranlagen sehr stabil. Deswegen werden diese auch zunehmend für die Detektion bzw. Feststellung von anthropogenen Einflüssen in Gewässern herangezogen. Ihre Effekte als Schadstoffe in der Natur sind Gegenstand aktueller Untersuchungen.

(iii) Nanomaterialien werden wegen ihrer unterschiedlichen Eigenschaften in verschiedensten Produkten verarbeitet. Nanosilber (nAg) stellt ein prominentes Beispiel dar. Es wird wegen seiner antimikrobiellen Wirkung zum Beispiel in medizinischen Bandagen oder Sportbekleidung eingearbeitet. Es zeichnet sich zunehmend Konsens darüber ab, dass Nanomaterialien geringe umweltrelevante Risiken aufweisen, wobei in vielen Bereichen noch weiterführende Untersuchungen und Daten fehlen.

(iv) Per- und polyfluorierte Alkylverbindungen umfassen mehr als 6000 Einzelsubstanzen, die über lange Zeit wegen ihrer hohen thermischen und chemischen Stabilität in unterschiedlichsten Produkten (Nahrungsmittelverpackun- 
gen, Hygieneprodukte, Farben etc.) verarbeitet wurden. Sie weisen eine hohe Transportfähigkeit in der Atmosphäre und Persistenz in der Natur auf, weshalb sie neben Trinkwasser mittlerweile rund um die Welt im Blut vieler entlegen lebender Tiere gefunden wurden. In der Europäischen Union ist die Erzeugung verboten und die Verwendung stark reglementiert.

(v) Pharmazeutika und Hormone gelangen aus anthropogenen Quellen über kommunale oder industrielle Kläranlagenabflüsse, urbane Regenwasserabflüsse oder Deponien in die Umwelt. Bei Fischen wurden unterschiedlichste Effekte, wie z.B. Änderungen im Verhalten oder der Morphologie beobachtet. In der Umwelt kommt es zur Akkumulation in Organismen und Sorption an Sedimenten. Durch weitere chemische Umwandlung können Umwandlungsprodukte mit teilweise unbekannten Wirkungen entstehen.

(vi) Nebenprodukte aus der Trinkwasser- und Schwimmbaddesinfektion (DBPs - disinfection by-products) stellen eine Konsequenz der Abtötung von Krankheitserregern und Mikroorganismen dar. Mehr als 700 Stoffe sind dokumentiert. Epidemiologische Studien liegen vor, welche die negativen Effekte auf den Menschen wie Blasenkrebs, Geburtenabbrüche oder Fehlbildungen belegen.

(vii) UV-Filter sind in Sonnencremen, Shampoos und anderen Kosmetikartikeln enthalten, um die Haut oder Haare vor schädlichen Sonnenstrahlen zu schützen. Sie gelangen direkt beim Schwimmen oder indirekt über das Abwassersystem in die Umwelt. Sie können durch Ähnlichkeiten zu Östrogen hormonelle Wirkung, Entwicklungstoxizität oder akute Toxizität aufweisen und stehen in enger Verbindung mit der Korallenbleiche in Meeren.

(viii) Bromierte Entflammungshemmer besitzen eine hohe Persistenz in der Umwelt und werden weiter in vielen Produkten wie Möbel oder Elektronikartikel eingesetzt, obwohl ihre Verwendung auch in einigen Ländern verboten ist. Auch neuere, bromfreie Entflam- mungshemmer weisen negative umweltrelevante Wirkungen auf.

(ix) 1,4-Dioxan wird als Lösungsmittel eingesetzt und wurde als möglicherweise kanzerogen für Menschen eingestuft und. Neue Untersuchungen legen nahe, dass die 1,4-Dioxan-Belastung des Trinkwassers von 30 Mio. Menschen in den USA über dem Gesundheitsschwellwert für Krebs $(0,35 \mu \mathrm{g} / \mathrm{l})$ liegt. Es ist sehr gut wasserlöslich, nicht flüchtig und adsorbiert schlecht an Aktivkohle, oxidiert nicht leicht und wird in Wasser auch nicht leicht biologisch abgebaut.

(x) Algen-Toxine wie z.B. Microcystine werden von Algen freigesetzt und können zu Fischsterben und Krankheiten bei Tieren und Menschen führen. Beispielsweise stehen sie in Zusammenhang mit Krebserkrankungen. Akute Gefährdungen wurden an verschiedenen Standorten bei Algenblüten dokumentiert.

(xi) Mikroplastik beschreibt Kunststoffe, die kleiner als $5 \mathrm{~mm}$ groß sind. Sie können von größeren Partikeln abbrechen oder direkt in dieser Größenordnung in z. B. Kosmetikprodukten verwendet werden und indirekt über die $\mathrm{Ab}$ wassersysteme in die Natur gelangen. Sie werden von Organismen wie Vögeln, Walen, Meeresreptilien oder Zooplankton aufgenommen und akkumulieren sich so. Es wird geschätzt, dass Menschen im Durchschnitt $5 \mathrm{~g}$ Kunststoff (das entspricht der Masse einer Kreditkarte) pro Woche aufnehmen.

Aufgrund landwirtschaftlicher Einträge sowie industrieller und kommunaler Kläranlagenabläufe kommen in Oberflächengewässern häufig Spuren organischer Stoffe, wie Arzneimittelrückstände, iodierte Röntgenkontrastmittel, Pflanzenschutzmittel und deren Metabolite, perfluorierte Verbindungen, Benzinzusatzstoffe (MTBE/ETBE) oder auch synthetische Komplexbildner vor. Vor allem persistente und polare, gut wasserlösliche organische Stoffe können selbst bei langer Untergrundpassage nicht ausreichend zurückgehalten werden (IAWR und IAWD 2007).

Aber auch eher unpolare Chemikalien wie polyzyklische aromatische Kohlenwasserstoffe oder chlorierte Kohlenwasserstoffe weisen mitunter eine hohe Persistenz auf und reichern sich dadurch in Schwebstoffen, Sedimenten sowie verschiedensten Organismen an. Weitere Schadstoffquellen sind Metallionen (Schwermetalle wie Kupfer, Zink, Blei, Cadmium und Halbmetalle wie Antimon oder Arsen), die in größeren Mengen beispielsweise in industriellen Rückständen der Erzverhüttung wie Schlacken vorkommen. Da diese als Recyclingstoffe für Uferverbauungen eingesetzt werden, kann es auch zu einem Eintrag während der Uferpassage kommen (Grathwohl et al. 2012).

Als wasserwerksrelevant werden Stoffe bezeichnet, die sich weitgehend persistent verhalten und bei der Bodenpassage nicht abgebaut werden (z.B. Tetrachlorethen, Atrazin oder Carbamazepin), während trinkwasserrelevante Stoffe (z. B. EDTA, MTBE und Amidotrizoat) persistent und nicht an Aktivkohle adsorbierbar sind (IAWR und IAWD 2007).

Der heutige Nachweis von organischen Spurenstoffen in den Gewässern kann auf die Entwicklung der analytischen Messtechnik zurückgeführt werden. Aufgrund der ständigen Verbesserung der Analysegeräte können selbst extrem niedrige Konzentrationen von Stoffen im Bereich Nanogramm pro Liter $\left(10^{-9} \mathrm{~g} / \mathrm{L}\right)$ nachgewiesen werden (IAWR und IAWD 2007).

Massenspektrometrie und Gas- oder Flüssigchromatographie bilden die Basis der modernen Analyse von organischen Spurenstoffen. Diese Geräte können einerseits dazu verwendet werden, gezielt ganz bestimmte Substanzen nach ihren Eigenschaften (Aufenthaltszeiten in der Chromatographie Säule, Molekülmasse oder Spektrum von Ionenmassen) zu bestimmen. Dies beschreibt die gezielte (targeted) Analyse. Andererseits kann der sogenannte ungezielte Ansatz verfolgt werden, um über den gesamten Messbereich von Masse und chromatographischen Aufenthaltszeiten Substanzen zu detektieren. Dieser Ansatz ermöglicht die gleichzeitige Untersuchung einer sehr großen Anzahl (>1000) von Substanzen. Die Herausforderung bei diesem Ansatz liegt daraus folgend in der Identifikation dieser Substanzen. Richardson und Kimura (2020) stellen fest, dass die ungezielte Analyse zurzeit einen sehr starken Trend in der Analyse von Spurenstoffen darstellt und dass von vielen Forschergruppen an Routinen und standardisierten Arbeitsabläufe für 
die Messung und Interpretation der Daten gearbeitet wird.

Laut EU-Wasserrahmenrichtlinie soll die effizienteste Maßnahmenkombination zur Reduktion von Spurenstoffen bei der Trinkwasseraufbereitung angewendet werden, wobei die Vermeidung des Eintrags in den Wasserkreislauf oberste Priorität hat. Obwohl Spurenstoffe aus heutiger Sicht kein akutes Problem darstellen, ist bezüglich des Wissens um deren Umweltauswirkungen, der Risikoeinschätzung für die Trinkwasserversorgung sowie der Maßnahmenentwicklung noch erheblicher Forschungsbedarf gegeben. Dazu zählen insbesondere die Bestimmung immissionsseitiger Anforderungen an Emissionsreduktionen, Weiterentwicklung praxistauglicher Systeme zur Aufklärung bestimmter Wirkungsmechanismen ausgewählter Substanzen in Stoffgemischen, die Verbesserung der Datengrundlage zur Bewertung von anthropogenen Spurenstoffen und ihren Abbauprodukten im Wasserkreislauf und des von ihnen ausgehenden Risikos für Lebewesen sowie die Ermittlung der Eintragspfade in das Abwassersystem, die Landwirtschaft und in die Gewässer mittels Stoffflussanalysen als Grundlage für Managementoptionen. Vor allem bei der Trinkwassergewinnung aus Uferfiltrat ist das Verhalten der Spurenstoffe in der Grenzschicht zwischen Oberflächengewässer und Grundwasser entscheidend, um beurteilen $\mathrm{zu}$ können, welche nachfolgenden Aufbereitungsschritte noch notwendig sind. Eine grundsätzliche Nachrüstung von Kläranlagen mit einer zusätzlichen Reinigungsstufe wie Ozonung oder Aktivkohle ist nach dem aktuellen Stand nicht notwendig (ÖWAV 2013).

Der Österreichische Wasser- und Abfallwirtschaftsverband (ÖWAV) weist auf die Bedeutung von Spurenstoffen als Herausforderung für die Wasserressourcen hin. Es wird eine Spurenstoffstrategie gefordert, welche die Quellen und Eintragswege der Stoffe sowie die Kosten und Wirksamkeit von Maßnahmen zur Reduktion der Emissionen berücksichtigt (ÖWAV 2020).

\subsection{Kosten}

Neben gesetzlichen und technischen Anforderungen spielen bei der Wahl des Wasseraufbereitungsverfahrens auch wirtschaftliche Aspekte eine entscheidende Rolle. Da sich die Faktoren Rohwasserqualität, Anlagengröße, -kapazi- tät und -auslastung, Chemikalienzugabe sowie Nachbehandlungserfordernisse von Ort zu Ort unterscheiden und folglich der Energieaufwand schwankt, ist eine Kostenschätzung schwer durchführbar. Generell betragen die gesamten Wasseraufbereitungskosten bei den typischen Verfahren immer weniger als $0,5 € / \mathrm{m}^{3}$, häufig auch weit unter $0,2 € / \mathrm{m}^{3}$. Aufwendige Techniken wie Nitratentfernung, Teilentsalzung oder Entkarbonisierung sind dementsprechend teurer. Zusätzlich zu berücksichtigen sind Aspekte des Betriebs wie der Personalaufwand, die Stabilität von Aufbereitungsprozessen bei schwankender Rohwasserqualität und die Sicherheit bei der Einhaltung der Grenz- und Richtwerte. Wässer mit relativ geringen Qualitätsschwankungen, wie Grundwässer und Uferfiltrate, bieten die Grundlage, um den Aufbereitungsprozess sicher automatisch oder halbautomatisch betreiben zu können (Jekel 2004).

Anhand einer Neukonzeption der Trinkwasserversorgung in der Region Vojvodina (Serbien) wurde mittels kleintechnischer Versuche bzw. Labormessungen gezeigt, dass zur Aufbereitung des aus der Theiß geförderten Uferfiltrats eine konventionelle Aufbereitung mit den Stufen Belüftung/Oxidation, Flockung, Filtration und Desinfektion ausreicht. Die spezifischen Kosten für die Bereitstellung von Trinkwasser aus diesem Uferfiltrat hätten bei schätzungsweise $0,1-0,2 € / \mathrm{m}^{3}$ (ohne Wasserverteilung) zuzüglich etwa $0,15 € / \mathrm{m}^{3}$ für den Bau und den Erhalt der Uferfiltratbrunnen betragen. Im Vergleich dazu resultierten bei der Aufbereitung von Tiefengrundwasser aufgrund dessen problematischer Zusammensetzung spezifische Aufbereitungskosten von bis zu $0,9 € / \mathrm{m}^{3}$, da erhöhte Dosiermengen an Chemikalien erforderlich wären. Die Abschätzung der gesamten Investitionskosten für die Trinkwasserversorgung der Region (Endausbau für rd. 230.000 Einwohner) mittels zweier Uferfiltratgewinnungen an der Theiß ergab 70 Mio. $€$ (durchschnittliche langfristige inkrementelle Kosten: $0,95 € / \mathrm{m}^{3}$ ), wovon die Anlagen für die Wasserverteilung und Speicherung den Hauptkostenfaktor bilden. Somit ergeben sich durch die Uferfiltratgewinnung gegenüber der Aufbereitung von Tiefengrundwasser erhebliche Kostenvorteile (Stauder et al. 2009).

Grooters (2007; zit. in Ahmed und Marhaba 2016) untersuchte die Anwen- dung von Uferfiltration als Vorbehandlungsstufe $\mathrm{zu}$ Umkehrosmoseanlagen an drei US-amerikanischen Flüssen. Bei Berücksichtigung von Kapital-, Betriebs- und Wartungskosten wurde ein Einsparungspotenzial von 10 bis $20 \%$ für den späteren Anlagenbetrieb festgestellt.

\section{Zusammenfassung}

Die Uferfiltration kann sowohl als Ressource wie auch als vorgeschaltete Methode bei der (Trink-)Wasseraufbereitung angesehen werden. Die mechanische Filtration, die Sedimentation, die Sorption und der mikrobiologische Abbau stellen die vier wesentlichen Mechanismen der Reinigungsprozesse dar. Es wurde dargestellt, welche Erkenntnisse über deren Wirkung auf partikuläre Wasserinhaltsstoffe, Mikroorganismen und Viren, Nährstoffe, Schwermetalle und Metallkationen und organische Wasserinhaltsstoffe vorliegen.

Auf partikuläre Stoffe wirken insbesondere Oberflächen- und Tiefenfiltration, wobei vor allem die Trübung als Summenparameter herangezogen werden kann. Es kann grundsätzlich von einer weitgehenden Entfernung partikulärer Stoffe bei der Uferfiltration ausgegangen werden.

Die Elimination, Reduktion sowie Inaktivierung von Mikroorganismen und Viren bei der Untergrundpassage geht auf Filtrierung (Protozoen, Cyanobakterien), Adsorption und biologischen $\mathrm{Ab}$ bau und Inaktivierung zurück. Bei sehr hohen Belastungen im Oberflächengewässer können auch coliforme Keime nicht immer vollständig entfernt werden. In diesen Fällen kann jedoch zumindest von einer sehr starken Reduktion ausgegangen werden.

In Bezug auf Nährstoffe stellen Ammonium, Nitrat und Phosphat in der Regel keine Probleme bei Uferfiltraten dar.

Das Entfernungspotenzial bei Schwermetallen und Metallkationen ist stark von den Milieubedingungen abhängig und generell hoch bei Eisen und Mangan, mäßig für Cadmium, Zink und Cyanid und gering bei Arsen oder Nickel. Bei der Reduktion und Elimination von Eisen und Mangan ist die Wirkung auf Sorption sowie Ionenaustausch und Fällungsprozesse zurückzuführen.

Bei der Verringerung organischer Wasserinhaltsstoffe wirken biologischer Abbau, Sorption aber auch die Mi- 
schung mit landseitigem Wasser. Die Milieubedingungen, vor allem aber der Sauerstoffbedarf, haben einen starken Einfluss. Die Summenparameter DOC und TOC zeigen, dass in den allermeisten Fällen eine effektive Reduktion durch Uferfiltration vorliegt. Der organische Anteil „neuer“ wasserrelevanter Stoffe (emerging pollutants) stellt zunehmend eine Herausforderung dar (Toxizität, Persistenz).

Insgesamt ist die Reinigungsleistung stark von den Redoxbedingungen, der Qualität des infiltrierenden Wassers und des natürlichen Grundwassers, der Fließstrecke und -dauer sowie von Bodenbeschaffenheit und Klimaverhältnissen abhängig.

Herausforderungen für die Uferfiltration ergeben sich einerseits aus den noch nicht detailliert darstellbaren Einflüssen durch den Klimawandel. Standortabhängig sind über Änderungen von Abflussregime und Temperatur jedoch Auswirkung auf Qualität und Quantität zu erwarten.

Andererseits führt der verstärkte Einsatz von Chemikalien, Pflegeprodukten, medizinischen Produkten und Ähnlichem zum Auftreten neuer Spurenstoffe in allen Bereichen des Wasserkreislaufs. Auch die Weiterentwicklung von Analysesystemen (Massenspektrometrie etc.) macht Substanzen in immer geringeren Konzentrationen bestimmbar, und so rücken bisher nicht berücksichtigte Stoffe in den Fokus von Untersuchungen. Aktuellen Entwicklungen um Nontargeted-Analyse und der Identifikation von Stoffen, die nicht erwartet aber detektiert wurden, stellen eine Strategie zum Umgang mit der schier unüberschaubaren Anzahl an Substanzen dar, welche in anthropogen beeinflussten Wasserkörpern auftreten. Aufgrund der gewonnenen Informationen in den Forschungsberichten ist ersichtlich, dass vor allem auch in Bezug auf das Wissen um die Umweltauswirkungen neuer Spurenstoffe, die Risikoeinschätzung für die Trinkwasserversorgung sowie die Maßnahmenentwicklung noch erheblicher Forschungsbedarf gegeben ist.

Nicht aus jedem Oberflächengewässer kann, selbst nach vorgeschalteter Bodenpassage, unter Berücksichtigung der Wirtschaftlichkeit Trinkwasser hergestellt werden. Besondere Herausforderungen stellen auch hier die neuen Wasserinhaltsstoffe (emerging pollutants), überhöhte Belastungen des Oberflächengewässers oder Nebenprodukte der Aufbereitungsverfahren dar. Trotzdem zeigen viele Studien, dass die Uferfiltration im Vergleich zu direkten Aufbereitungsverfahren von Wasser aus Oberflächengewässern eine effektive Methode insbesondere auch zur Reduktion der Kosten darstellt.
Funding Open access funding provided by University of Natural Resources and Life Sciences Vienna (BOKU).

Open Access Dieser Artikel wird unter der Creative Commons Namensnennung 4.0 International Lizenz veröffentlicht, welche die Nutzung, Vervielfältigung, Bearbeitung, Verbreitung und Wiedergabe in jeglichem Medium und Format erlaubt, sofern Sie den/die ursprünglichen Autor(en) und die Quelle ordnungsgemäß nennen, einen Link zur Creative Commons Lizenz beifügen und angeben, ob Änderungen vorgenommen wurden.

Die in diesem Artikel enthaltenen Bilder und sonstiges Drittmaterial unterliegen ebenfalls der genannten Creative Commons Lizenz, sofern sich aus der Abbildungslegende nichts anderes ergibt. Sofern das betreffende Material nicht unter der genannten Creative Commons Lizenz steht und die betreffende Handlung nicht nach gesetzlichen Vorschriften erlaubt ist, ist für die oben aufgeführten Weiterverwendungen des Materials die Einwilligung des jeweiligen Rechteinhabers einzuholen.

Weitere Details zur Lizenz entnehmen Sie bitte der Lizenzinformation auf http://creativecommons.org/licenses/ by/4.0/deed.de.
Ahmed, A.K.A., Marhaba, T.F. (2016): Review on river bank filtration as an in situ water treatmen process. Clean Techn Environ Policy 19, 349-359 (2017). https://doi.org/10.1007/s10098-0161266-0

Botzenhart, K. (1998): Verhalten von Bakterien und Viren bei der Aquiferpassage. HTW Dresden (Geotechnik \& Wasserwesen), TU Dresden (Institut f. Grundwasserwirtschaft), Universitä Leipzig (Institut für Geophysik und Geologie), UFZ Leipzig-Halle: Tagungsband zum Workshop „Uferfiltrat“. Dresden

Brauch, H.-J., Sacher, F, Denecke, E., Tacke, T. (2000): Wirksamkeit der Uferfiltration für die Entfernung von polaren organischen Spurenstoffen. gwf Wasser/Abwasser 141, Nr. 4, S. 226-234

Chorus, I., Klein, G., Rotard, W. (1995): Beeinträchtigung des Uferfiltrates verschieden belasteter Berliner Gewässer durch biogene Stoffe und die Auswirkung von Sanierungsmaßnahmen. Deutsche Forschungsgemeinschaft: Schadstoffe im Grundwasser. Bd. 3. Weinheim: VCH, S. 181-205

Clayton (1995): Biogeochemical processes in the Torgau aquifer adjacent to the River Elbe

Dash, R., Mehrotra, I., Kumar, P., Grischek T. (2008): Lake bank filtration at Nainital, India: water-quality evaluation. Hydrogeol J. 16:1089-1099
Dash, R.R., Prakash, E.B., Kumar, P., Mehrotra, I., Sandhu, C., Grischek, T. (2010): River bank filtration in Haridwar, India: removal of turbidity, organics and bacteria. Hydrogeol J 18:973-983 Davis, J.A. (1982): Adsorption of natural dissolved organic matter at the oxide/water interface Geochim. Cosmochim. Acta 46, S. 2381-2393

Diem, S., Cirpka, O.A., Schirmer, M. (2013): Modeling the dynamics of oxygen consumption upon riverbank filtration by a stochastic-convective approach. J Hydrol 505:352-363 Doussan, C., Ledoux, E., Detay, M. (1998): River-Groundwater Exchanges, Bank Filtration, and Groundwater Quality: Ammonium Behavior. J. Environ. Qual. 27, S. 1418-1427

Driescher, E., Gelbrecht, J., Stellmacher, R. (1989): Phosphat im unterirdischen Grundwasser. 2. Mitteilung: Zum Verhalten von Phosphat bei der Uferfiltration. Acta hydrophys. 33, Nr. 2/3, S. 75-101

Eckert, P., Lamberts, R., Wagner, C. (2008): The impact of climate change on drinking water supply by riverbank filtration. Water Sci Technol 8(3):319-324

Ellis, K. V. \& Aydin, M.E. (1995). Penetration of solids and Biological Activity in to Slow Sand Filters. Water Research. 29(5):1333-1341.

Förstner, U. (1995): Redoxeinflüsse auf die Bindungsform und Mobilität von Spurenmetallen im Untergrund. Deutsche Forschungsgemein schaft: Schadstoffe im Grundwasser. Bd. 3. Weinheim: VCH, S. 309-391

Gerlach, M. (1998): Zur Bedeutung von $\mathrm{Hu}-$ minstoffen bei der Trinkwassergewinnung aus Uferfiltrat. Rheinisch-Westfälisches Institut für Wasserforschung GmbH: Berichte (Dissertationen) aus dem IWW. Bd. 22. Duisburg

Ghodeif, K, Grischek, T, Bartak, R, Wahaab, R., Herlitzius, J. (2016): Potential of river bank filtration (RBF) in Egypt. Environ Earth Sci 75:1-13

Grathwohl, P., von Schlippenbach, U., Gimbel, R., Hillemeier, B., LIBRA, J., Meckenstock, R., Reetz, K., Schubert, H., Ternes, T. (2012): Wasserbeschaffenheit, in: Hüttl, R. F., Bens O. (2012): Georessource Wasser - Herausforderung Globaler Wandel. Beiträge zu einer integrierten Wasserressourcenbewirtschaftung in Deutschland (acatech STUDIE), Springer Verlag, Heidelberg u. a., 158-226

Grooters (2007): Role of riverbank filtration in reducing the costs of impaired water desalination. Desalination and water purification research and development. Program report no. 122 Haberer, K., Drews, M., Waltenberg, M.-C. (1984): Entfernung organischer Wasserinhaltsstoffe bei der Langsamsandfiltration. Vom Wasser 63, S. 121-140 
Hiscock, K.M., Grischek, T. (2002): Attenuation of groundwater pollution by bank filtration. J Hydrol 266:139-144

Hofmann, T. \& Schöttler, U. (1998): Microparticle facilitated transport of contaminants during artificial groundwater recharge. In: Peters et al. (eds.): Artificial recharge of Groundwater, Balkema, Rotterdam, 205-210.

Hoppe-Jones, C., Oldham, G., Drewes, J.E. (2010): Attenuation of total organic carbon and unregulated trace organic chemicals in US riverbank filtration systems. Water Res 44:4643-4659 IAWR, IAWD (2007): Position der IAWR und IAWD zu Spurenstoffen in den Gewässern, Statement, 28. März 2007, Köln

Jardine, P.M., Weber, N.L., McCarthy, J.F. (1989): Mechanisms of Dissolved Organic Carbon Adsorption on Soil. Soil Sci. Soc. Am. J. 53, S. 1378-1385

Jekel, M. (2004): Anforderungen an Trinkwasser und an die Trinkwasseraufbereitung, in: Gimbel, R., Jekel, M., Liessfeld, R. (2004): Wasseraufbereitung - Grundlagen und Verfahren, Lehr- und Handbuch Wasserversorgung Bd. 6, Oldenbourg Industrieverlag, München, 1-22

Jekel, M., \& Haberer, K. (2004): Entfernung von anorganischen Mikroverunreinigungen und von Radionukliden, in: Gimbel, R., Jekel, M., Liessfeld, R. (2004): Wasseraufbereitung-Grundlagen und Verfahren, Lehr- und Handbuch Wasserversorgung Bd. 6, Oldenbourg Industrieverlag, München, 551-587

Lahti, K., Silvonen, J., Kivimäki, A. L. \& Erkomaa, K. (1996): Removal of cyanobacteria and their hepatotoxins from raw water in soil and sediments columns. Proceedings of an International Symposium on Artificial Recharge of Groundwater, Finnish Environment Institute, Helsinki, Finland, 187-195

Lee, J.-H., Hamm, S.-Y., Cheong, J.-Y., Kim, H.S., Ko, E.-J., Lee, K.-S., Lee, S.-I. (2009): Characterizing riverbank-filtered water and riverwater qualities at a site in the lower Nakdong River basin. Repub. Korea J Hydrol 376:209-220

Massmann, G., Nogeitzig, A., Taute, T., Pekdeger, A. (2008): Seasonal and spatial distribution of redox zones during lake bank filtration in Berlin. Ger Environ Geology 54:53-65

Mikels, M.S. (1992): Characterizing the Influence of surface water on water produced by collector wells (PDF). J-Am Water Works Assoc. 84:77-84

ÖWAV (2013): Anthropogene Spurenstoffe in de aquatischen Umwelt, ÖWAV-Positionspapier Wien

ÖWAV (2020): Positionen und Forderungen der Wasserwirtschaft in Österreich 2020, ÖWAV-Positionspapier, Wien
Preuß, G., Nehrkorn, A. (1988): Mikrobielle Sukzessionen im Grundwasser bei der Uferfiltration-Veränderungen in Dichte und Verteilung verschiedener Bakteriengruppen. Z. dt. geol. Ges. 139, S. 575-586

Preuß, G. \& Schulte-Ebbert, U. (2000): Artificial Groundwater Recharge and Bank filtration. In Rehm HJ, Reed G, editors. Biotechnology. Vol. 11c. Weinheim: Wiley-VCH; 2000. pp. 425-444.

Quanrud, D.M., Arnold, R.G., Wilson, L.G., Gordon, H.J., Graham, D.W., Amy, G.L. (1996): Fate of organics during column studies of soil aquifer treatment. J. Env. Engineering 122, Nr. 4 S. 314-321

Richardson, S. D. \& Kimura, S. Y. (2020): Water Analysis: Emerging Contaminants and Current Issues. Analytical Chemistry 2020, 92, 1, 473-505 Schachtschabel, P., Blume, H.-P., Brümmer, G., Hartge, K.H., Schwertmann, U., u. a.(1998) Lehrbuch der Bodenkunde (Scheffer/Schachtschabel), 14. Aufl. Stuttgart: Enke Verlag, -3-43284774-2

Schmoller, C. (2014): Uferfiltration - Stand der Technik und neue Herausforderungen für die weiterführende Wasseraufbereitung an Rhein und Donau, Masterarbeit am Institut für Siedlungswasserbau, Industriewasserwirtschaft und Gewässerschutz, Wasservorsorge, Department für Wasser-Atmosphäre-Umwelt, Universität für Bodenkultur Wien.

Schöttler, U. (1995): Neuere Techniken der Grundwasseranreicherung. In: Verein zur Förderung des Instituts der Wasserversorgung, $\mathrm{Ab}$ wasserbeseitigung und Raumplanung der $\mathrm{TH}$ Darmstadt (Hrsg.): Grundwasseranreicherung Stand der Technik und Entwicklung-- Schriftenreihe WAR 83, Darmstadt

Schöttler, U. (1986): Wechselwirkungen von ausgewählten Spurenelementen mit chemischbiologischen Prozessen bei Uferfiltration, künstlicher Grundwasseranreicherung und Untergrundpassage. Veröffentlichungen des Instituts für Wasserforschung $\mathrm{GmbH}$ Dortmund Nr. 34. Dortmund. - ISSN 0342-474 X

Schubert, J. (2000): Entfernung von Schwebstoffen und Mikroorganismen sowie Verminderung der Mutagenität bei der Uferfiltration. gwf Wasser/Abwasser 141, Nr. 4, S. 218-225

Schulte-Ebbert, U. (2004): Künstliche Grundwasseranreicherung und Untergrundpassage, in: Gimbel, R., Jekel, M., Liessfeld, R. (2004): Wasseraufbereitung - Grundlagen und Verfahren, Lehr- und Handbuch Wasserversorgung Bd. 6, Oldenbourg Industrieverlag, München, 403-439

Shamrukh, M., Abdel-Wahab, A. (2011): Water pollution and riverbank filtration for water supply along river Nile, Egypt. Riverbank filtration for water security in desert countries. Springer, Dordrecht, pp 5-28

Singh, P., Kumar, P., Mehrotra, I., Grischek, T. (2010): Impact of riverbank filtration on treatment of polluted river water. J Environ Manage 91:1055-1062

Sontheimer, H. (1991): Trinkwasser aus dem Rhein?St. Augustin: Academia Verlag, - 3-88345381-1

Sontheimer, H., Jekel, M., Roberts, P.V. (1981): Probleme der Wasserqualität bei Verwendung von aufbereitetem Abwasser zur Grundwasseranreicherung. Aurand, K. (Hrsg.): Bewertung chemischer Stoffe im Wasserkreislauf. Berlin: Erich Schmidt Verlag, S. 136-153

Sprenger, C., Lorenzen, G., Hülshoff, I., Grützmacher, G., Ronghang, M., Pekdeger, A. (2011): Vulnerability of bank filtration systems to climate change. Sci Total Environ 409:655-663

Stauder, S., Geiges, M., Kühn, W. (2009): Neukonzeption der Trinkwasserversorgung unter Berücksichtigung neuartiger Verfahren zur Aufbereitung alkalischer, stark arsen- und huminstoffhaltiger Grundwässer am Beispiel der Städte Becej, Novi Becej, Kikinda und Zrenjanin in de Region Vojvodina (Serbien), Schlussbericht, Technologiezentrum Wasser, Karlsruhe Stuyfzand, P. J. \& Kooiman, J. W. (1996): Elimination of pollutants during artificial recharge and bank infiltration: a comparison. International Symposium on Artificial Recharge of Groundwater, 3-5 June 1996, Helsinki, Finland. Wang, J.Z. (2005): American experience with riverbank filtrationsystem-A detailed case study at Louisville Kentucky. J Harbin Inst Technol (New Series) 12:75-81

Wegener, G., Persin, J., Karrenbrock, F., Rörden, O., Hübner, I. (1999): Vorkommen und Verhalten von natürlichen und synthetischen Östrogenen und deren Konjugate in der aquatischen Umwelt. Vom Wasser 92, S. 347-360 Weiss, W.J., Bouwer, E.J., Aboytes, R., LeChevallier, M.W., O'Melia, C.R., Le, B.T., Schwab, K.J. (2005): Riverbank filtration for control of microorganisms: results from field monitoring. Water Res 39:1990-2001

Ziegler, D. H. (2001):Untersuchungen zur nachhaltigen Wirkung der Uferfiltration im Wasserkreislauf Berlin, Dissertation an der Fakultät III

- Prozesswissenschaften der Technischen

Hinweis des Verlags Der Verlag bleibt in Hinblick auf geografische Zuordnungen und Gebietsbezeichnungen in veröffentlichten Karten und Institutsadressen neutral. 